University of Nebraska - Lincoln

DigitalCommons@University of Nebraska - Lincoln

USDA Wildlife Services - Staff Publications

U.S. Department of Agriculture: Animal and Plant Health Inspection Service

2021

\title{
Effects of social structure and management on risk of disease establishment in wild pigs
}

\author{
Anni Yang \\ Colorado State University - Fort Collins, yangann1@colostate.edu \\ Peter E. Schlichting \\ University of Georgia, pschlich@srel.uga.edu \\ Bethany Wight \\ University of Florida \\ Wesley M. Anderson \\ University of Florida \\ Sarah M. Chinn \\ University of Georgia \\ Follow this and additional works at: https://digitalcommons.unl.edu/icwdm_usdanwrc \\ Part of the Natural Resources and Conservation Commons, Natural Resources Management and

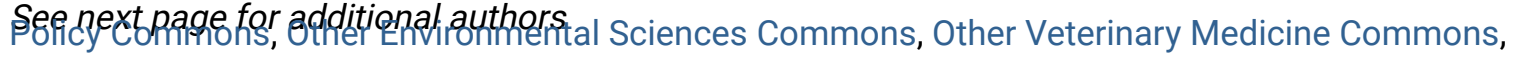 \\ Population Biology Commons, Terrestrial and Aquatic Ecology Commons, Veterinary Infectious Diseases \\ Commons, Veterinary Microbiology and Immunobiology Commons, Veterinary Preventive Medicine, \\ Epidemiology, and Public Health Commons, and the Zoology Commons
}

Yang, Anni; Schlichting, Peter E.; Wight, Bethany; Anderson, Wesley M.; Chinn, Sarah M.; Wilber, Mark Q.; Miller, Ryan S.; Beasley, James C.; Boughton, Raoul; Vercauteren, Kurt C.; Wittemyer, George; and Pepin, Kim M., "Effects of social structure and management on risk of disease establishment in wild pigs" (2021). USDA Wildlife Services - Staff Publications. 2442.

https://digitalcommons.unl.edu/icwdm_usdanwrc/2442

This Article is brought to you for free and open access by the U.S. Department of Agriculture: Animal and Plant Health Inspection Service at DigitalCommons@University of Nebraska - Lincoln. It has been accepted for inclusion in USDA Wildlife Services - Staff Publications by an authorized administrator of DigitalCommons@University of Nebraska - Lincoln. 


\section{Authors}

Anni Yang, Peter E. Schlichting, Bethany Wight, Wesley M. Anderson, Sarah M. Chinn, Mark Q. Wilber, Ryan S. Miller, James C. Beasley, Raoul Boughton, Kurt C. Vercauteren, George Wittemyer, and Kim M. Pepin 


\title{
Effects of social structure and management on risk of disease establishment in wild pigs
}

\author{
Anni Yang ${ }^{1,2}$ (D) | Peter Schlichting ${ }^{3}$ | Bethany Wight ${ }^{4}$ | Wesley M. Anderson ${ }^{4}$ | \\ Sarah M. Chinn ${ }^{3}$ | Mark Q. Wilber ${ }^{5}$ (D) | Ryan S. Miller ${ }^{6}$ (D) James C. Beasley ${ }^{3}$ (D) | \\ Raoul K. Boughton ${ }^{4}$ | Kurt C. VerCauteren ${ }^{2}$ | George Wittemyer ${ }^{1}$ (D) | Kim M. Pepin ${ }^{2}$
}

\begin{abstract}
${ }^{1}$ Department of Fish, Wildlife, and Conservation Biology, Colorado State University, Fort Collins, CO, USA; ${ }^{2}$ National Wildlife Research Center, United States Department of Agriculture, Animal and Plant Health Inspection Service, Fort Collins, CO, USA; ${ }^{3}$ Savannah River Ecology Laboratory, Warnell School of Forestry and Natural Resources, University of Georgia, Aiken, SC, USA; ${ }^{4}$ Wildlife Ecology and Conservation, Range Cattle Research and Education Center, University of Florida, Ona, FL, USA; ${ }^{5}$ Ecology, Evolution and Marine Biology, University of California, Santa Barbara, Santa Barbara, CA, USA and ${ }^{6}$ Center for Epidemiology and Animal Health, United States Department of Agriculture, Animal and Plant Health Inspection Service, Veterinary Service, Fort Collins, CO, USA
\end{abstract}

Correspondence

Anni Yang

Email: yangann1@colostate.edu

\section{Funding information}

USDA-APHIS National Feral Swine Damage Management Program; US Department of Energy

Handling Editor: Thierry Boulinier

\section{Abstract}

1. Contact heterogeneity among hosts determines invasion and spreading dynamics of infectious disease, thus its characterization is essential for identifying effective disease control strategies. Yet, little is known about the factors shaping contact networks in many wildlife species and how wildlife management actions might affect contact networks.

2. Wild pigs in North America are an invasive, socially structured species that pose a health concern for domestic swine given their ability to transmit numerous devastating diseases such as African swine fever (ASF). Using proximity loggers and GPS data from 48 wild pigs in Florida and South Carolina, USA, we employed a probabilistic framework to estimate weighted contact networks. We determined the effects of sex, social group and spatial distribution (monthly home-range overlap and distance) on wild pig contact. We also estimated the impacts of managementinduced perturbations on contact and inferred their effects on ASF establishment in wild pigs with simulation.

3. Social group membership was the primary factor influencing contacts. Betweengroup contacts depended primarily on space use characteristics, with fewer contacts among groups separated by $>2 \mathrm{~km}$ and no contacts among groups $>4 \mathrm{~km}$ apart within a month.

4. Modelling ASF dynamics on the contact network demonstrated that indirect contacts resulting from baiting (a typical method of attracting wild pigs or game species to a site to enhance recreational hunting) increased the risk of disease establishment by $\sim 33 \%$ relative to direct contact. Low-intensity population reduction ( $<5.9 \%$ of the population) had no detectable impact on contact structure but reduced predicted ASF establishment risk relative to no population reduction.

5. We demonstrate an approach for understanding the relative role of spatial, social and individual-level characteristics in shaping contact networks and predicting 
their effects on disease establishment risk, thus providing insight for optimizing disease control in spatially and socially structured wildlife species.

\section{KEYWORDS}

African swine fever, contact structure, management, network modelling, wild pig

\section{1 | INTRODUCTION}

Contact among individuals plays a fundamental role in infectious disease transmission, affecting disease persistence, spread and outbreak severity (Craft, 2015). Thus, identifying how factors like social structure, space use and individual-level characteristics affect host contact rates has been-and remains-a critical goal in disease ecology (Silk et al., 2017). The advent of proximity loggers in wildlife studies coupled with theoretical developments in epidemiology have highlighted that population-level assumptions about contact often ignore individual-level heterogeneities that affect disease dynamics (Bansal et al., 2007). To address this challenge, contact networks, rather than mean contact rates, are often inferred for host populations and the resulting estimates are used to parameterize transmission models that more realistically inform disease/wildlife management (Craft, 2015; Silk et al., 2017). While contact networks used in these types of studies are invariably a result of biological processes, rarely do studies elucidate the mechanisms underlying the pairwise contact that generated the observed network structure (White et al., 2017), which is important for targeting control strategies effectively. Instead, descriptive metrics including degree, betweenness and transitivity centrality, are often used, and statistical models are then applied to model the factors influencing those metrics (Boehm et al., 2009; Reynolds et al., 2015; Weber et al., 2013). However, descriptive metrics only represent contact features of the observed sample, and this approach may not detect the mechanisms underlying formation of a contact network. An alternative approach is to statistically infer contact networks using probabilistic inference from pairwise contacts (Welch et al., 2011; Wilber et al., 2019). By probabilistically modelling pairwise contacts based on covariates, we can better understand the underlying mechanisms of contact processes and propagate their uncertainty more thoroughly when the network is scaled up to a larger population (Welch et al., 2011). Covariatebased approaches for probabilistically inferring contact networks have rarely been applied to wildlife diseases (White et al., 2017), despite their utility for disentangling how biological factors shape contact networks.

Social structuring into family groups is common in wildlife populations from small-group species such as raccoons Procyon lotor or wild pigs Sus scrofa, to large-group species like elk cervus canadensis (Sah et al., 2018). Such clustering introduces contact heterogeneity as individuals within groups contact each other more often than individuals in different groups (Drewe et al., 2011). How individuals interact can influence disease spread through a population, and individual heterogeneities of the contact network may affect an animal's risk of becoming infected (Silk et al., 2017). Contacts can also result from space sharing among individuals determined by their movement in response to resource availability (Best et al., 2014). Spatial distribution of the hosts, thus, has important implications for disease transmission given higher chance of contact among individuals sharing more space (Craft, 2015).

Wild pigs in North America are an invasive species, descended from domestic pigs and Eurasian wild boar and are also referred to as wild hogs, feral swine or feral hogs. Females occur in family groups with one to several adult females and offspring while adult males live independently. They often pose a health concern for domestic swine given their ability to transmit numerous devasting diseases like African swine fever (ASF; Miller et al., 2017). Previous studies suggested that social structure is one of the key factors determining contact heterogeneities in wild pigs (Podgórski et al., 2014, 2018). Wild pigs maintain matrilineal, multigenerational social groups of female adults with their offspring (Dardaillon, 1988; Podgórski et al., 2014). Group structure is dynamic with natal dispersal of males and some females at reproductive maturity and fission when groups become large or individuals temporarily join adjacent groups (Gabor et al., 1999; VerCauteren et al., 2020). Spatial overlap among groups is another important factor influencing wild pig contact (Pepin et al., 2016), and local and sub-population interactions are hypothesized to influence contact structure and disease transmissions among wild pigs (Cowled \& Garner, 2008). However, the interplay of social and spatial processes on contact networks which determine disease transmission remain poorly understood in wild pigs (Beasley et al., 2018), or in socially structured wildlife species in general (Sah et al., 2018).

Wildlife management tactics can also affect host movement and space use, which can in turn affect contact networks and disease spread (Donnelly et al., 2003). Culling of badgers Meles meles aimed at limiting bovine tuberculosis (bTB) transmission subsequently disrupted badger social structure and thus increased badger movement and bTB transmission to cattle (Donnelly et al., 2003). Multiple management techniques, including baited trapping, toxicants and culling, are currently being developed and used to control wild pigs/ boars (Boadella et al., 2012; Snow et al., 2019), and these techniques have been found to affect their movement and space use (BastilleRousseau et al., 2020; Fattebert et al., 2017). Baiting could affect direct and indirect contacts among wild pigs, particularly across social groups. Altering population density directly through management (culling/hunting) can also affect disease spread in wild pigs due to changes in contact structure. While these pathways have been 
assumed to impact transmission probabilities, quantification of their impacts is lacking.

Here we use proximity loggers and Global Positioning System (GPS) data from wild pigs to: (a) develop and apply an approach that estimates a weighted contact network, (b) infer the relative role and interactions among factors that shape wild pig contact, especially social structure, spatial processes, and sex and (c) examine how management-induced perturbations, namely removal and baiting strategies, affect contact structure and ASF establishment risk $\left(R_{0}\right)$.

\section{2 | MATERIALS AND METHODS}

\section{1 | Study area}

Our study area includes two sites: a $\sim 245 \mathrm{~km}^{2}$ area within the US Department of Energy's Savannah River Site (SRS) in South Carolina and the northeastern section of Archbold Buck Island Ranch (ABIR) in Florida. SRS is a $780-\mathrm{km}^{2}$ National Environmental Research Park with $\sim 68 \%$ of habitat consisting of upland pine forest and $22 \%$ comprised of bottomland hardwood forest (Imm \& McLeod, 2005). ABIR is a $42.3-\mathrm{km}^{2}$ beef cow-calf operation ranch managed at commercial production levels supporting an onsite agroecology research centre. ABIR runs $\sim 3,000$ head of cattle and consists of a mosaic of natural and altered habitats including pastures, oak-palm hammock forests and wetlands (Swain et al., 2013). Both sites support numerous and diverse wildlife species, including wild pigs. Wild pig density at SRS ranges $\sim 0.91-2.60$ adult pigs $/ \mathrm{km}^{2}$ (Keiter et al., 2017) with the adult density of our focal areas as 1.39 per $\mathrm{km}^{2}$ (Bastille-Rousseau et al., 2020). Adult wild pig density at ABIR ranges $\sim 1.90-2.95$ per $\mathrm{km}^{2}$ (P. Schlichting, unpub. data).

\subsection{Data collection and processing}

Wild pig capture and handling (Supporting Information 1) were conducted under approved University of Georgia IACUC protocol A2015 05-004 and A2015 12-017 at SRS and under University of Florida IACUC protocol 201808495 at ABIR. At SRS, we captured and deployed both proximity loggers (Sirtrack $\odot$ ) and GPS collars (Telonics TGW4501 and TGW4600) on 22 adult wild pigs (13 female [F] and 9 male [M]) from February 1 to November 15, 2017. The captured wild pigs covered most social groups in the focal study site, so at least one individual in those groups was sampled (given pre-collaring camera surveys). At ABIR, we captured and deployed both proximity loggers and GPS collars (Catlog GPS device and Lotek LMRT3 VHF Collars, Lotek (C) on 26 wild pigs (20F and 6M) from April 8, 2017 to June 5. However, due to GPS collar damage and battery exhaustion, only 38 animals (ABIR: 14F, 6M; SRS: 11F, 7M) had both proximity logger and GPS data available. We conducted baiting and removal tactics to manage wild pigs at SRS. We set up nine baiting stations with proximity loggers from July to August 2017 and continuously removed wild pigs (see removal intensity in Table S1) from September 14 to November 15, 2017.

Proximity loggers from both sites were programmed with a separation time of $255 \mathrm{~s}$ and began recording contacts at a 5-m distance. We did pre-field tests on proximity loggers to establish confidence in these threshold settings. GPS collars at SRS and ABIR recorded fixes at 1-hr and 30-min intervals respectively. Social group membership of wild pigs with GPS collars were assigned based on their spatial distributions (Figure S1). Wild pigs with home range (HR) overlap greater than $50 \%$ were classified in the same social group (calculation of HR detailed below; Gabor et al., 1999). Group membership of wild pigs with damaged GPS collars were assigned based on capture dates and pre-collaring camera surveys. Given different sampling schemes and data availability at two sites, we employed different subsets of data to address different research questions (Figure S2).

We combined proximity logger data between each unique pair of loggers that were recorded within a 2-min amalgamation window and then removed the remaining one-second contacts (Drewe et al., 2012). We considered a direct contact to occur if the logger of either individual in the unique wild pig pair recorded a contact. As wild pigs alter space use frequently due to shifts in resource availability and depletion and their reproductive phenology is less cyclic than wild boar (Keuling et al., 2009; Mayer \& Brisbin, 2009), we analysed data on a monthly scale to understand how the ever-changing reproductive and foraging behaviours might affect contact structure. In the analyses to assess effects of management processes, we also aggregated data according to the time periods of the management processes (baiting or culling) being assessed (Table S1). An indirect contact occurred when two wild pigs contacted the same baiting site within 5 days. Thus, indirect contacts were only recorded during the baiting period (SRS only) and we ignored other potential locations of indirect contact. We chose this 5-day interval to reflect the potential time that virulent strains of ASF virus remain infectious in the environment (Davies et al., 2017).

\section{3 | Modelling probabilistic weighted networks}

A previous study developed a method for inferring contact structure across species that included direct and indirect contact mechanisms within a single network (Wilber et al., 2019). This approach estimated the probability that two individuals (nodes) make contact (edges) within a given time frame. Her, we extended the previous framework to also estimate the number of contacts between nodes (edge weights) using a negative binomial (NB) hurdle model (McDowell, 2003). The NB hurdle model includes a hurdle component to estimate the probability that the contact occurred between two individuals and a NB component to model the contact rates (number of contacts over time), given that the contact occurred. The hurdle component could be a binomial or multinomial process depending on the number of possible contact modes. Our approach is an extension of dyadic independent Exponential Random Graph Model (Welch et al., 2011), which captures probabilities of 
edge formation and weight under the assumption that edges are independent after accounting for node- and edge-level covariates.

Consider a contact network of wild pig population that includes $c$ modes of contact (e.g. direct and indirect contact) and a no contact state (nc), meaning any given wild pig pair could experience $c+1$ possible contact states. The $k$ th observation of the hurdle component was defined by a dummy vector $\mu_{k}$ (zero and one represents the absence and occurrence of a type of contact, respectively) with $c+1$ possible contact states that a unique dyad composed of individual $m$ and $n$ from social group $i$ and $j$, respectively, could experience during the time interval of interest. If any modes of contact occurred between two pigs, a NB model was applied to capture the contact rates (W).

Hurdle component:

$$
\begin{gathered}
\mu_{k} \sim \text { Multinomial }\left(N_{k}, p_{1}, p_{2}, \ldots, p_{c}, p_{n c}\right)\left(\text { or } \mu_{k} \sim \operatorname{Binomial}\left(1, p_{1}, p_{n c}\right)\right), \\
\log \left(\frac{p_{g}}{p_{n c}}\right)_{k}=X^{g} \beta^{g}+\alpha_{m, j}^{g}+\alpha_{n, i}^{g} ; 1 \leq g \leq c .
\end{gathered}
$$

NB component:

$$
\begin{gathered}
W_{g}\left(\mu_{k}[g]=1\right) \sim \text { Negative Binomial }\left(\lambda_{g}, q_{g}\right) ; 1 \leq g \leq c, \\
\log \left(\lambda_{g}\right)=\log (\text { offset })+X^{g \prime} \beta^{g \prime}+\alpha_{m, i}^{g}{ }^{\prime}+\alpha_{n, j}^{g}{ }^{\prime},
\end{gathered}
$$

Priors:

$$
\begin{gathered}
\alpha_{m, i}^{g}, \alpha_{m, i}^{g}{ }^{\prime} \sim N\left(0, \sigma_{i}^{2}\right), \\
\sigma_{i} \sim \operatorname{HalfNormal}(0,4), \\
\beta^{g}, \beta^{g \prime} \sim N(0,4), \\
q^{g} \sim \operatorname{uniform}(0,50) .
\end{gathered}
$$

Parameter $N_{k}$ represents the number of different contact modes that the kth pair of animals experienced over the time interval $\left(1 \leq N_{k} \leq c\right) . g$ is the contact mode type. $X$ and $X^{\prime}$ are the matrices of covariates that affect the contact probability and rates respectively. Parameters $\beta$ and $\beta^{\prime}$ are the coefficients of these covariates. Parameters $\alpha$ and $\alpha^{\prime}$ are the social group-level random effects (with variation $\sigma_{i}^{2}$ ) in the NB and hurdle components of the model, respectively, which allows individuals $m$ and $n$ belonging to the same group $(i=j)$ to have more similar contact patterns than two individuals from other groups (i.e. we assume the behaviour of individuals in the same group is correlated; Podgórski et al., 2014). We set regularizing normal priors on all $\beta$ and $\beta^{\prime}$ coefficients of all variables, a uniform prior for $q$ (dispersion parameter in the NB distribution) and a half-normal prior for the variance of the social group-level random effects $\left(\sigma_{i}^{2}\right)$.

\subsection{Social effect on contact networks}

We used the wild pig data at SRS before management (Feb-Jun; 12F, 7M) and ABIR (Apr-Jun; 20F, 6M) to address the effects of social group and sex on wild pig direct contact. The covariates we included in these models were group_pair, sex_pair, month, overlap_time, site and number_of_pairs. The variable group_pair is a factor with two levels indicating whether the pair is from the same family groups (within- or between-group). The variable sex_pair is a three-level factor where levels represent the sex of each pig in contact pair $m$ and $n$; efor example, an ' $F \_M$ ' level represents female encountering male. We considered the number of days within each predefined time window (Table S1) that the pair of proximity loggers was active concurrently as a covariate, overlap_time, in the hurdle component and as an offset in the NB component adjusting for counts of contact over variable time periods. The number_of_pairs gives a vector of the number of unique wild pig pairs with proximity loggers available during each time window. The covariate month is a factor with levels representing the month in which contacts occurred. The variable site is a factor with two levels representing the study sites. All variables were screened for multicollinearity (Pearson's correlation coefficient $|r| \geq 0.7$; Vatcheva et al., 2016; Figure S3). The most informative variable was included when a strong correlation was detected among a set of variables.

\section{5 | Spatial effects on contact networks}

We used wild pigs from both sites (SRS: 8F, 6M; ABIR: 15F, 4M) with more than 1-month of GPS data available before baiting and removal to address the effects of spatial proximity on direct contacts. We considered two measurements to describe the spatial distribution of each wild pig pair: HR distance and overlap. To match the time window over which we aggregated the proximity data and given that contact events are time-specific (i.e. transmission window is not indefinite), we focused on monthly HR and employed the $a$ method Local Convex Hull (LoCoH) analysis (Getz et al., 2007) to estimate the 95\% HRs for each pig using the тьосон R-package (Lyons et al., 2013). The HR overlap (HRover) of two individual pigs was estimated using the intersection of the two HRs divided by the union of them (Lyons, 2014). HR distance (HRdist) was measured as the distance between medians of the locations of two wild pigs. Since we observed that some animals changed group membership during the study, we assigned social group membership monthly to each individual using the changes in monthly HR overlap (Figure S1). We incorporated the above spatial metrics as two additional covariates in the NB hurdle model to estimate the effects of spatial proximity on contact (Table S3).

\section{6 | Management effects on contact network and disease establishment}

We used data from SRS (Feb-Nov; 13F, 9M) to estimate the effects of management on wild pig contacts. Given indirect contacts were introduced via bait piles, we considered two contact types, direct and bait-mediated (indirect) contact and fitted a 
similar NB hurdle model with a multinomial hurdle component to the data. We tested the effects of removal with four different covariates: monthly number of pigs removed from the whole population (removed_pigs; Table S1), monthly number of collared pigs removed (removed_sample; Table S1), monthly cumulative proportion of wild pigs removed (cumulative_proportion; $5.9 \%$ in Sep, $7.8 \%$ in Oct, $8.6 \%$ in Nov) and monthly cumulative proportion of all pigs removed that were collared pigs (cumulative_sample_proportion; $5.2 \%$ in Sep, $11.1 \%$ in Oct, $23.5 \%$ in Nov). We used a binary variable (bait) to estimate if contacts occurred within the baiting period or not. Additionally, we included group pair, sex_pair, overlap_time and number_of_pairs to capture other factors and added another random effect of month to consider seasonal variation.

We fitted all NB hurdle models via Bayesian Markov chain Monte Carlo (MCMC) methods using JAGS v4.3.0 (Plummer, 2003) and JAGsUI R-package (Kellner, 2015). We ran 3 MCMC chains for 50,000 iterations, with thinning rate of 40 and burn-in of 10,000 iterations. We ensured convergence of the model and sufficient burn-in using Gelman-Rubin statistic (where $\hat{R}<1.05$ for convergence) for all coefficients and visual inspection of the trace plots (Gelman \& Rubin, 1992). We generated all additive combinations of covariates and compared the fitted model performance using widely applicable information criteria (WAIC; Gelman et al., 2014). To assess predictive accuracy, we simulated our best fit network models and compared the predicted network statistics (node-level degree and strength centrality) to observed and random network statistics (Supporting Information 2). We focused on degree (number of different individuals an animal connects to) and strength (number of contacts the animal makes with others over time), instead of other relevant metrics, because those two have strong impacts on disease dynamics, can be applied widely in different types of disease transmission frameworks (not only network models), and are most commonly examined (i.e. for comparison of results to other work). Also, they directly reflect the performance of the two common components of disease transmission models: contact probability (binomial component) and rates (NB component). We, hereafter, use 'contact occurrence/probability' and 'contact rates' to describe the features of a pairwise contact and network terminology 'degree' and 'strength' to describe a contact network predicted from the hurdle models. We followed the criteria and methods above for model selection and evaluation in all sets of analyses.

We used the predicted contact networks to evaluate the effects of contact degree and management on the relative likelihood of ASF establishment using the basic reproduction number, $R_{0}$, as a metric (Supporting Information 3). $R_{0}$ measures the expected number of secondary cases caused by an infectious host in a completely susceptible population (Dietz, 1993), with absolute values above 1 indicating that a pathogen can deterministically invade a population. Our estimate of ASF-specific, population-level $R_{0}$ was derived from an epidemiological model following ASF epidemiological processes and parameterized using the ASF-related parameters (e.g. transmission/death rate) and the predicted average degree (average number of other animals they connect to) across different time frames (Supporting Information 3). We also estimated the individual-level $R_{0}$ using node-level metrics to capture contact network heterogeneity, since disease spread depends on the hierarchy that infectious individuals arise in the network. We considered removal as an additional death rate other than natural and ASF-related death. Because our derivation of $R_{0}$ required specifying some unknown parameters (e.g. transmission probability given contact), we did not estimate absolute values of $R_{0}$. Rather, we rescaled the $R_{0}$ values to a baseline value based on different objectives (here a maximum individual-level $R_{0}$ over the study period was used as a baseline, so relative $R_{0}$ ranged from 0 to 1 ) to show how different management strategies change $R_{0}$ values relative to values from unweighted networks over the study period. Thus, our results cannot be used to identify disease invasion thresholds, rather they are relevant to determining the magnitude of effects of different conditions on disease invasion rates. We also calculated the sex-specific contribution to relative $R_{0}$ over the study period using the top-selected NB hurdle (multinomial) model.

To estimate the effects of management-induced perturbations on ASF transmission, we estimated the relationships between $R_{0}$ changes and the number of effective bait sites or removal rates. Although nine bait sites were placed at SRS, only two of them were located in the HRs of collared pigs during the study period. Thus, we only focused on effects of two bait sites on indirect contact. We randomly dropped indirect contacts introduced by each of the two bait sites and fitted the NB hurdle model with two contact modes and covariates including group_pair, sex_pair, overlap_time, bai, and number_of_pairs. We computed $R_{0}$ relative to non-baiting direct contacts as described above for scenarios with zero, one or two bait sites. Given the low removal intensity during the experimental removal treatment ( $5.9 \%$ of pigs removed), we examined possible effects of additional removal intensities by randomly dropping individuals from the collared population with a rate ranging from $10 \%$ to 80\% (2-20 removed individuals; 8 scenarios), which we ignored the unknown behavioural responses to different removal intensities. As wild pig contact varies across seasons given their response to resource availability, we also simulated the scenarios on removing wild pigs in different seasons with different contact degrees. We divided the observed data into a high-degree scenario (Feb-Apr), low-degree scenario (May-Jun) and with indirect contacts included (Jul-Aug; total of $3 \times 8$ scenarios). For each data-dropping scenario, we conducted 100 replicate simulations, fitted the NB hurdle model with group_pair, sex pair, overlap_time and number_of_pairs, and controlled monthly variations (month excluded). We then calculated average degree for each scenario and used them to compute $R_{0}$ relative to non-removal. We focused on the impact of removal intensity on average degree, using both the data we had and by extrapolation beyond it to predict the removal intensity that would be required to drastically impact average degree. All analyses were implemented in R v3.5.3 (R Core Team, 2019). 


\section{3 | RESULTS}

\section{1 | Social effect on contacts}

We selected the more parsimonious (Model 1.1 in Table S2) of two models with similar performance (other model: Model 1.2; Figure 1a,b) to estimate social effects on contact structure. This model predicted the observed node-level degree and strength distributions better than random networks (Figure S4; Figure 2). Figure 3 shows the observed contact networks in different time windows at two study sites.

Social group membership and sex affected wild pig contacts in both sites (Table S2; Figures 1a,b and 2). As expected, in the contact (a)

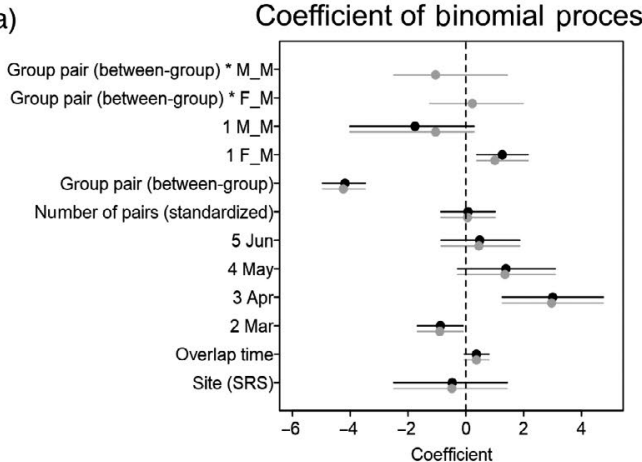

(c)

Coefficient of binomial process

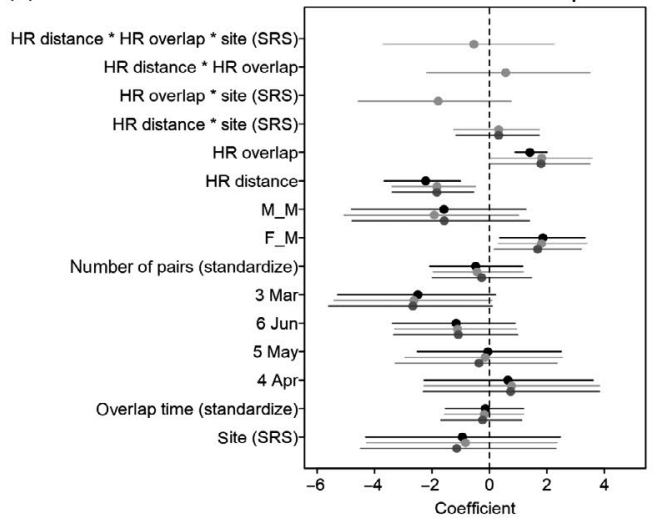

(e)

Coefficient of multinomial process (direct)

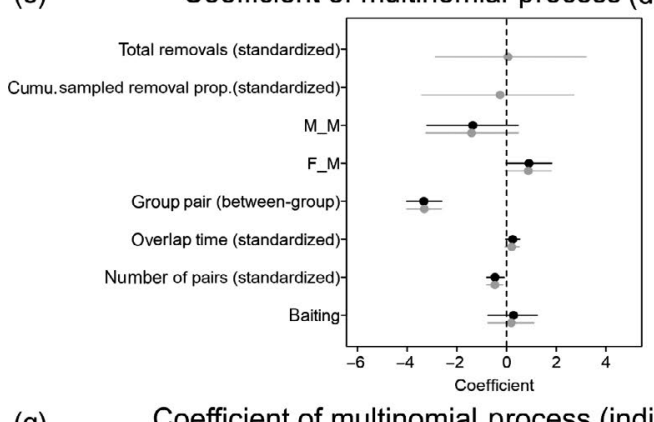

(g) Coefficient of multinomial process (indirect)

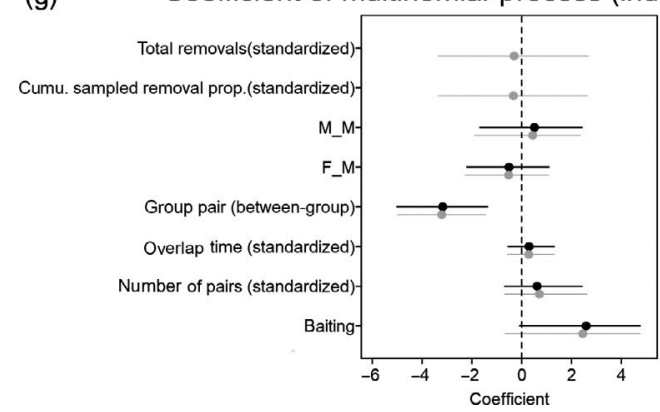

(b)

Coefficient of neaative binomial process

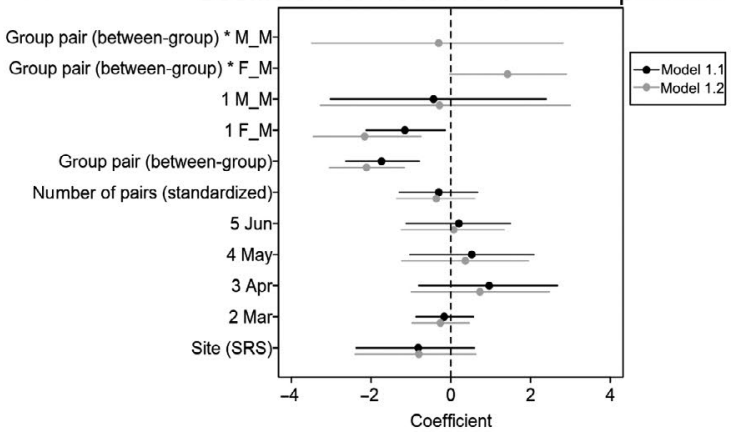

(d)

Coefficient of neaative binomial process

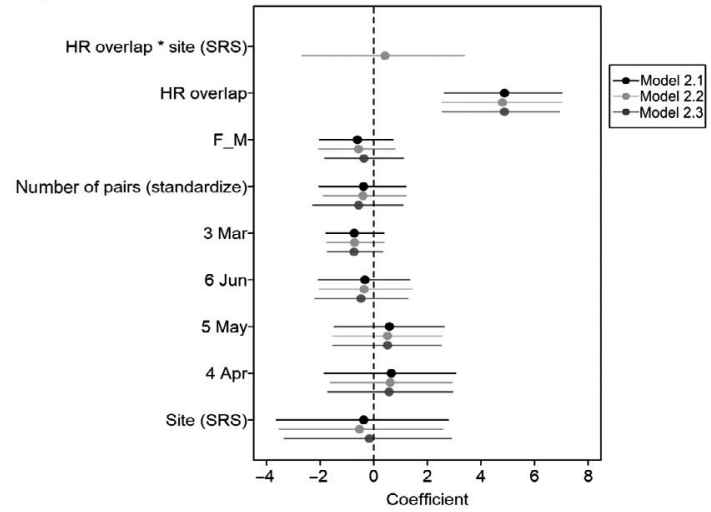

(f) Coefficient of neaative binomial process (direct)

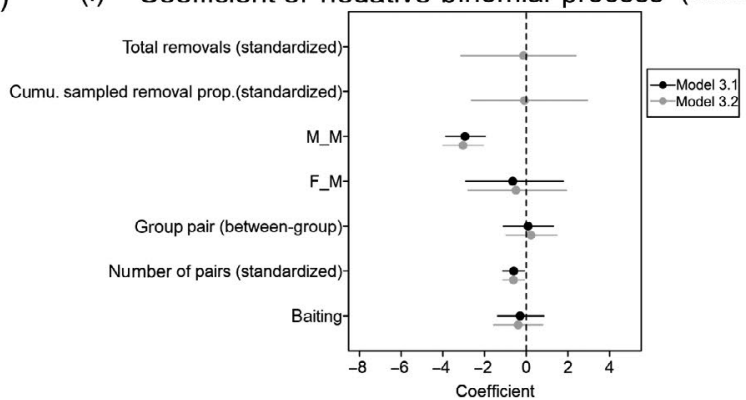

(h) Coefficient of neaative binomial process (indirect)

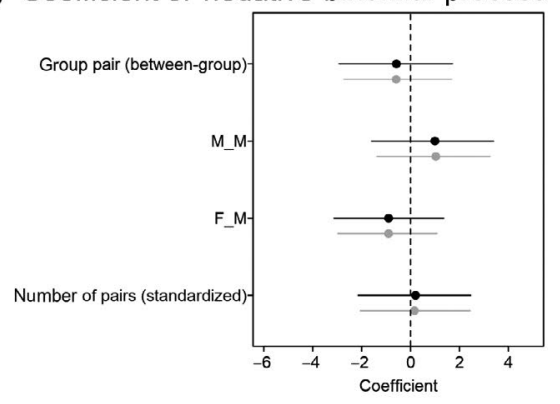

FIGURE 1 Coefficient estimates and 95\% credible interval from the negative binomial (NB) hurdle models identifying the effect of social structure and sex (a, b), spatial proximity (c, d), and management (e-h) on contact structure. For the month factor, February is the baseline. For group pair factor, within-group is the baseline. For the sex pairs, female encountering female $\left(F_{-} F\right)$ is the baseline. In each set of panels, models in black are top-selected, while models in grey are the competing models 

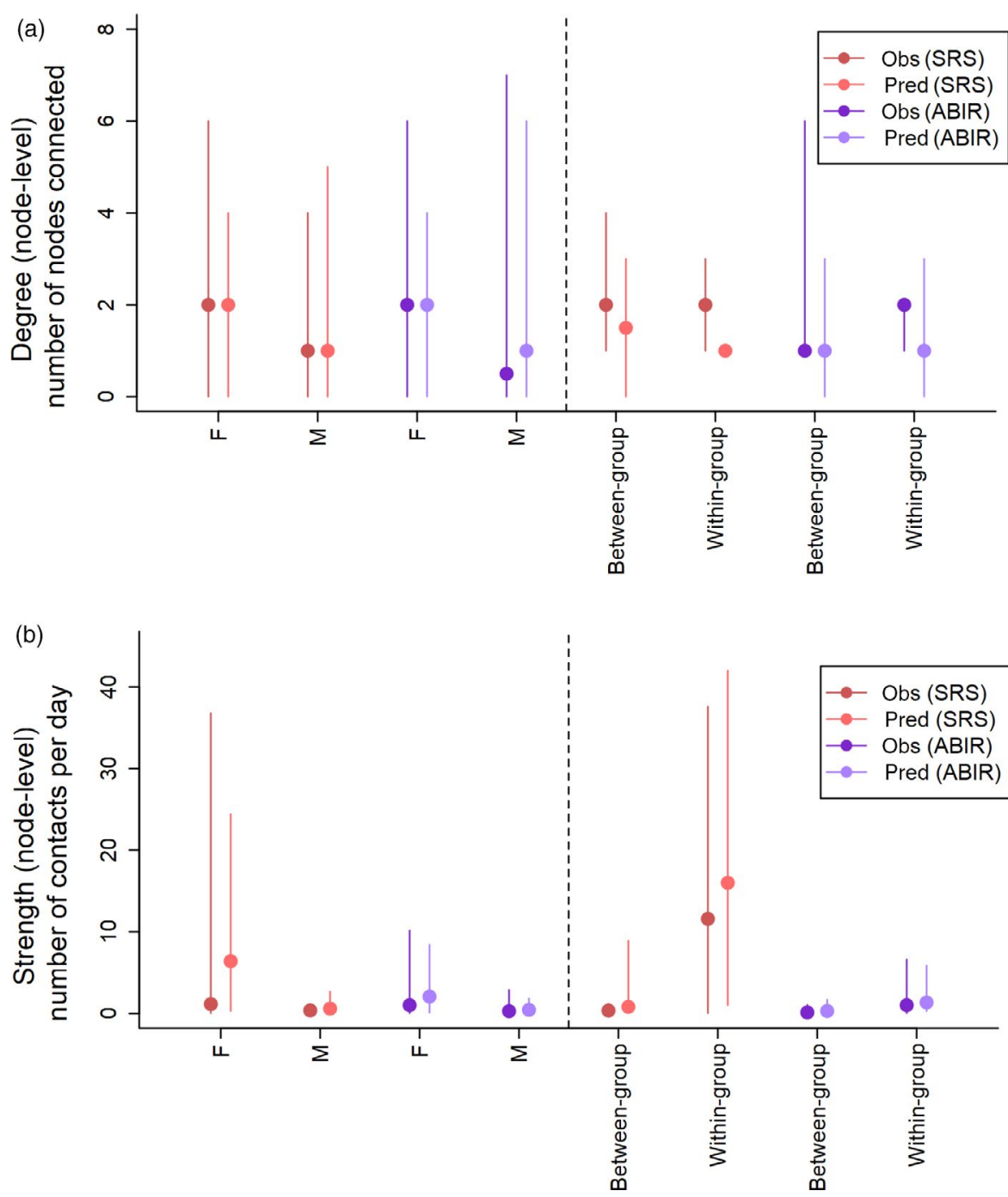

FIGURE 2 Predicted and observed (a) degree and (b) strength centrality across females, males and group memberships depict high within-group direct contact rates and sex-specific contact variation. The error bars show the median and $95 \%$ quantile of the node-level degree or strength in a subpopulation network, within-group strength centrality was 10 times higher than between-group strength at SRS, and it was doubled relative to between-group strength at ABIR. Female adults tended to contact more individuals (higher degree) and have higher strength, while male adults contacted fewer unique individuals but with large variation in degree distributions.

\section{2 | Spatial effect on contacts}

Similarly, Model 2.1 was selected to describe the effects of spatial proximity (Table S3; Figure 1c,d), predictive accuracy of which also performed better than random networks (Figure S5). Monthly HR overlap increased contact probability and contact rates, as expected (a proxy for group membership). Contact probability decreased when monthly HR distance increased; most direct contacts occurred when monthly HR distances were $<2 \mathrm{~km}$, and no contacts occurred when monthly HR distances were $>4 \mathrm{~km}$ (Figure 4). Individuals with monthly HR distances within $1 \mathrm{~km}$ often contacted more than once per day.

\subsection{Management effects on contacts and ASF establishment}

We selected Model 3.1, the more parsimonious model without removal-related covariates to evaluate management effects, compared to a competing model with similar performance (i.e. Model 3.2; Table S4), suggesting that low-intensity removal $(<5.9 \%$ of total population) did not impact the contact networks. Model predictions of degree and strength across different contact types performed better than random networks (Figures S6 and S7). The addition of baiting sites increased overall contact rates and average degree by introducing a mechanism for indirect contact, but baiting showed no impact on direct contact (Figures $1 \mathrm{e}-\mathrm{h}$ and 5 ).

Before baiting and removal (Feb-Jun), relative $R_{0}$ for ASF naturally (without perturbations) decreased as average degree decreased (Figure 6a). When baiting began (Jul-Aug), the relative $R_{0}$ increased due to the addition of indirect contact and remained high during the first 2 weeks in Sep after baiting had ceased. Relative $R_{0}$ then decreased from 0.08 immediately before the removal period to 0.03 in Sep, when the number of removed pigs reached $\sim 5.9 \%$ of the total population 
FIGURE 3 Observed unweighted contact network in different time windows at Savannah River Site (SRS) and Archbold Buck Island Ranch (ABIR)
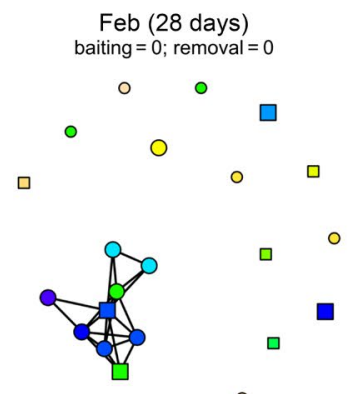

0

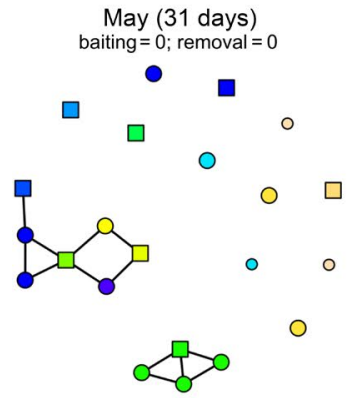

Aug (31 days)

baiting $=1$; removal $=0$
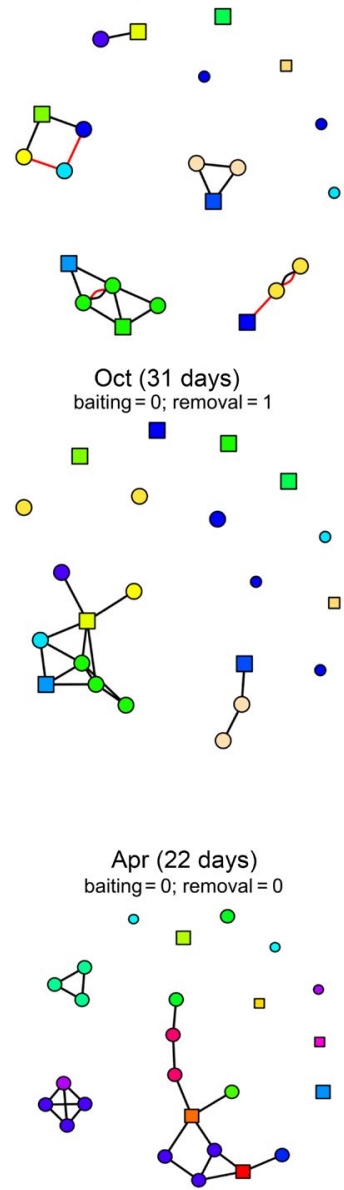

\section{SRS site}

Mar (31 days)

baiting $=0$; removal $=0$

Apr (30 days)

baiting $=0$; removal $=0$

- 0

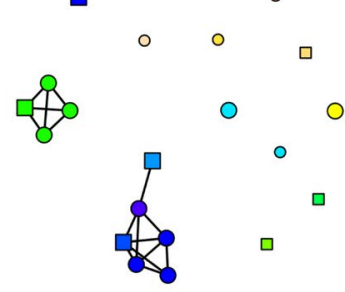

$\square$

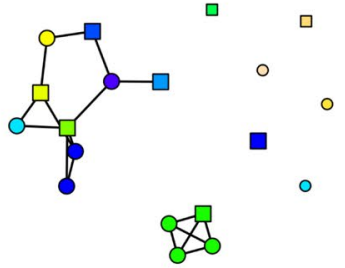

Jun (30 days)

baiting $=0$; removal $=0$
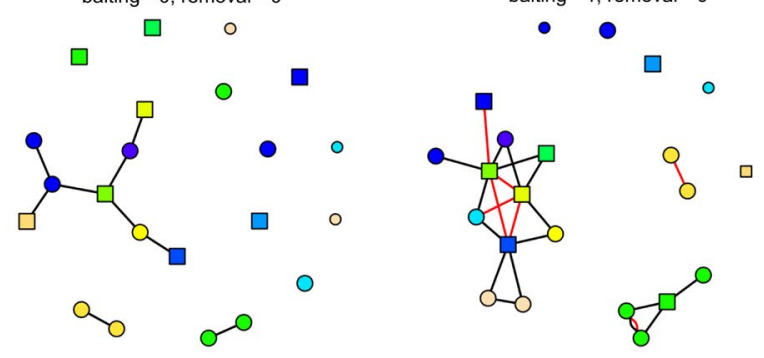

$\circ$

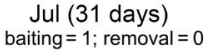

Sep (13 days)

baiting $=0$; removal $=0$

○ $\square \quad 0$

baiting $=0$, removat $=1$

00

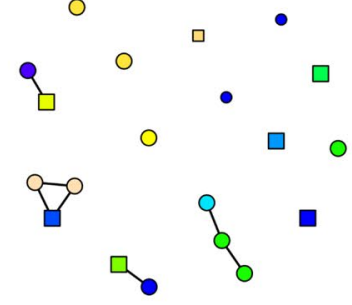

$\square$

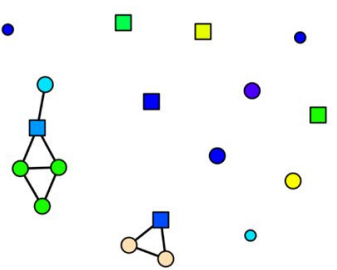

Nov (15 days)

baiting $=0$; removal $=1$

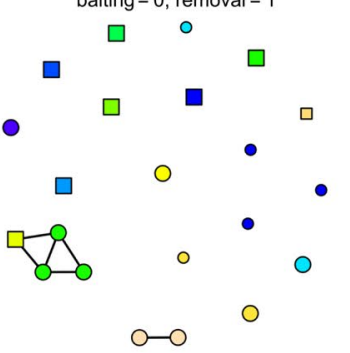

Legend

Nodes

$\square$ Male with active proximity logger

$\square$ Male with inactive proximity logger

Female with active proximity logger

$\bigcirc$ Female with inactive proximity logger Color represents group membership

Edges

- Direct contact

- Indirect contact

\section{ABIR site}
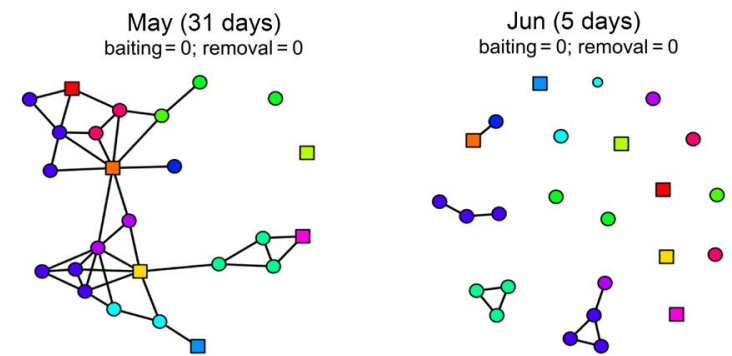

in the study site. Thus, although low-intensity removal did not alter the contact structure, it reduced the relative $R_{0}$ by $\sim 42 \%-78 \%$ in mid Sep-Nov due to the additional reduction of host density (death rates underpin $R_{0}$ but not contact degree). The contributions of females and males to the relative $R_{0}$ of $\mathrm{ASF}$ followed the trend of their contributions to average contact degree, with females contributing more (Figure 6b). $R_{0}$ increased by $\sim 27 \%$ with one bait site and by $\sim 33 \%$ with two bait sites relative to before-baiting direct contact (Figure $6 \mathrm{c}$ ). In the 

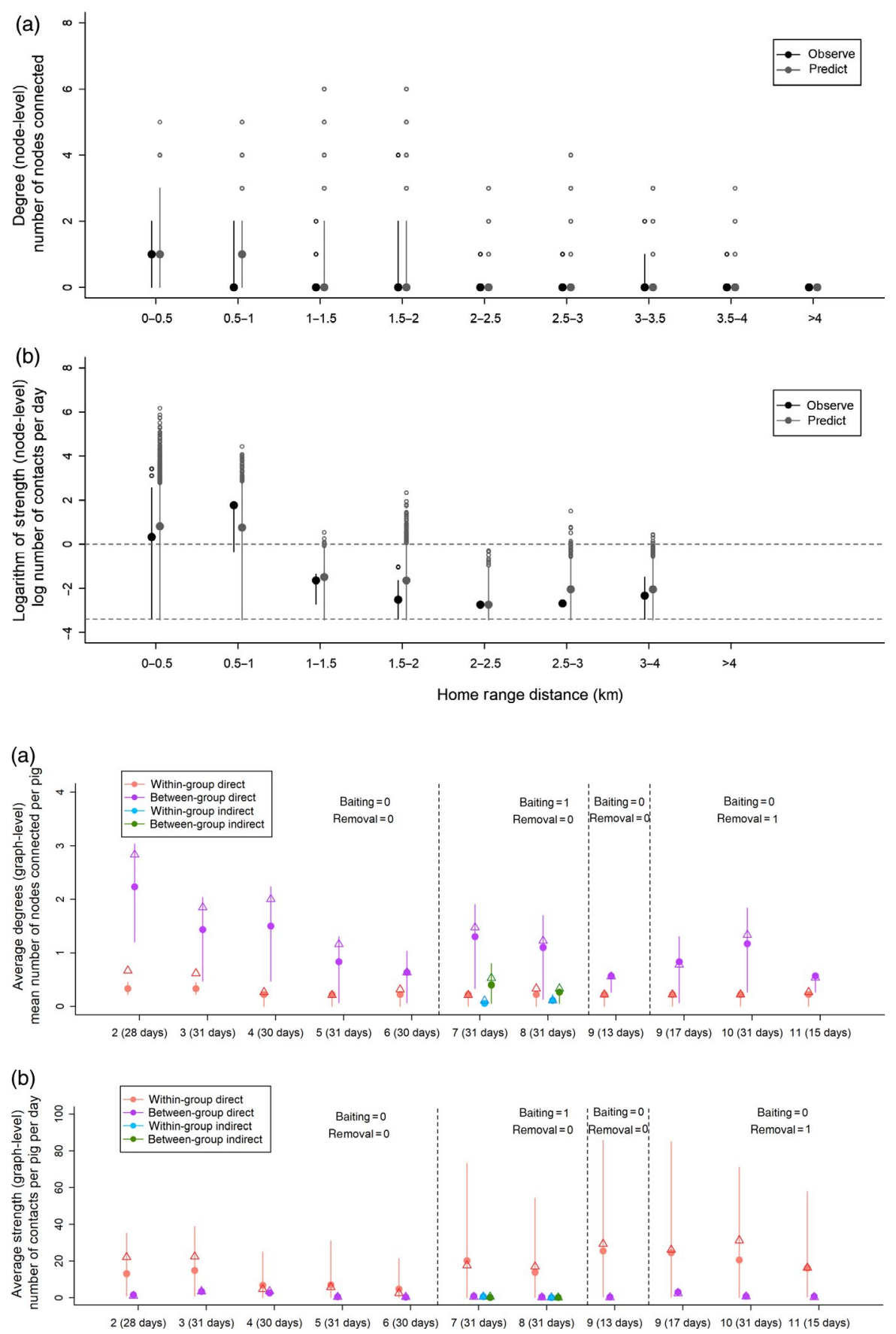

FIGURE 4 Predicted and observed (a) degree and (b) strength centrality (log scaled) in different categories of home-range distances. Error bars show the median and $95 \%$ quantile of the node-level degree or strength in a subpopulation. Contacts are rare $>2 \mathrm{~km}$ and non-existent $>4 \mathrm{~km}$
FIGURE 5 Predictions of graph-level (a) degree and (b) strength centrality for within-group direct contacts, betweengroup direct contacts, within-group indirect contacts and between-group indirect contacts before baiting and removal, and during the baiting and removal periods. Triangles are the observations. Error bars show the median and $95 \%$ quantile of the graph-level predicted degree or strength in each time frame. Indirect contacts were introduced by baiting effects but did not alter direct contact degree and strength simulated scenarios, removing $10 \%$ of the sampled population reduced the $R_{0}$ relative to zero-removal by $\sim 50 \%-70 \%$ (similar to the experimental treatment) depending on the contact degree in a population (Figure $6 \mathrm{~d}$ ). $R_{0}$ of the low-degree population was more sensitive to removal than that of the population with high-degree contact or indirect contacts included.

\section{4 | DISCUSSION}

Disease emergence and transmission in widespread, invasive species is of critical concern globally given its potential to disrupt ecological processes, threaten food production systems and impact human health (Crowl et al., 2008). Fundamental to developing management approaches to contain and remove disease threats is characterizing the processes that underlie the transmission of disease. Here we implemented a probabilistic approach to define weighted contact networks across two wild pig populations with two main features (contact probability and rate) to understand the effects of management processes on transmission of ASF, a disease of global concern.

\subsection{Factors influencing direct contact}

As found in related systems (Podgórski et al., 2018), withingroup strength centrality of the wild pig contact network was 
FIGURE 6 (a) Population-level relative $R_{0}$ for African swine fever (ASF) virus under the monthly changes in predicted (black) and observed (red) contact structures over the study period. The grey areas are the upper and lower boundaries of individual-level relative $R_{0}$. (b) Sexspecific contributions to population-level relative $R_{0}$. (c) Effects of the number of baits on ASF population-level $R_{0}$ relative to direct contacts before baiting. Error bars show 95\% quantiles. (d) Effects of monthly removal rates on population-level $R_{0}$ relative to non-removal in simulated scenarios. Error bars show the upper and lower boundaries of individual-level relative $R_{0}$
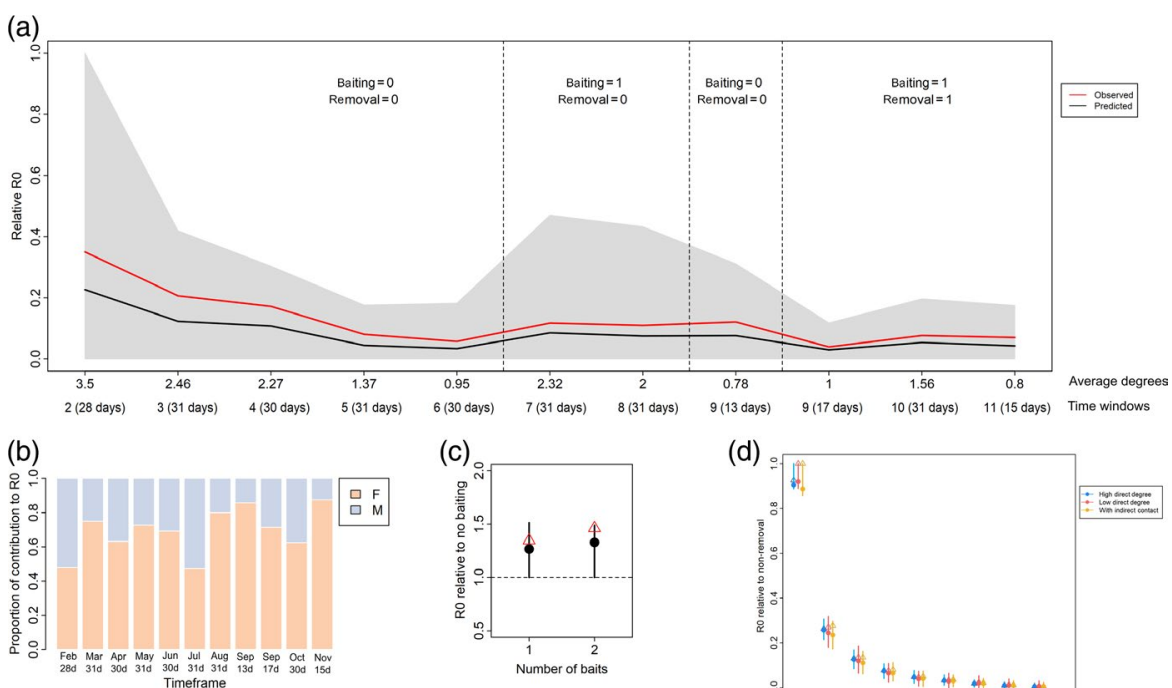

much higher than between-group contacts likely due to the high group cohesion and the philopatry of females. Since the spatial extent of disease spread is driven by contacts between groups, this social structuring might introduce a constraint on disease transmission (Hirsch et al., 2013). Considering the low betweengroup strength in the contact networks, we might expect limited spread of highly virulent pathogens such as ASF virus. However, wild pigs at both sites had relatively large variances in betweengroup contact degree, suggesting pathogens have the potential to be transmitted broadly throughout the population. The high within-group contact rates could enable persistence by leading to enough new cases to sustain transmission of ASF in the rarer between-group contacts. There is similar evidence of social structure effect in other wildlife disease systems. For example, studies in wild meerkats Suricata suricatta (Drewe et al., 2011), primates (Ryan et al., 2013) and badgers (Weber et al., 2013) suggested that clustered contact networks due to social and demographical factors limited disease transmission across population. These results could be explained by network modularity, a metric describing the strength of division of a network into different clusters, which is often negatively correlated with disease spread (Girvan \& Newman, 2002). Although modularity was not evaluated here, by examining the effect of group membership on network metrics, we were able to quantify group effects on contact probability and rate, providing a mechanistic understanding of modularity effects on the network.

Most contacts occurred among individuals within a 2-km monthly HR distance with high contact rates, and there were no contacts with monthly HR distances $>4 \mathrm{~km}$ (maximum observation), which is similar to previous studies in wild pigs/boars (Pepin et al., 2016; Podgórski et al., 2018) and other ungulates (Grear et al., 2010). Additionally, contact strength decreased gradually as HR distance among individuals increased. These results indicated that spatial constraints on animal movement and contact network have the potential to limit the spatial spread of diseases in this system (Cowled \& Garner, 2008). The spatially constrained contact pattern may interact with social constraints to slow disease spread and establishment as most of the high-rate contacts within a 2-km monthly HR distance are within-group contacts. Thus, our findings suggest a $2-\mathrm{km}$ culling radius around a disease detection might be sufficient to capture most ongoing transmission as long as the detection is rapid relative to the onset of infectiousness. However, these thresholds for monthly HR distance might vary in other study sites based on population density, landscape features and resource availability; for example, ASF virus may spread farther and faster in a denser population or in populations with larger home ranges, thus requiring a larger culling radius.

\section{2 | Factors influencing indirect contact}

Food contamination can be a major source of infectious disease transmission by facilitating indirect contact, as shown for brucellosis, ASF and foot-mouth disease (Bates et al., 2003; Cross et al., 2007; Sánchez-Vizcaíno et al., 2013). To spatially constrain hosts, supplemental feeding is provided to wildlife populations to administer vaccines or create spatial separation between species of concern and susceptible hosts (Cross et al., 2007; Sokolow et al., 2019). However, bait piles or feed can increase contacts (directly and indirectly) among individuals, potentially increasing disease prevalence in wildlife populations (Cross et al., 2007). Despite only two bait piles being used by wild pigs here, the overall contact degree and the ASF establishment risk were increased substantially by increasing indirect contact between individuals from different social groups. Besides artificial feeding for wildlife, other food sources including livestock supplements and shared natural forage/water locations (not considered here) could also serve to enhance contact and, thereby, disease transmissions (Brahmbhatt et al., 2012; Wilber et al., 2019). Indirect contacts were estimated here based on a fixed ASF virus decay rate of 5 days. However, the survival of ASF virus varies depending on environmental conditions, for example, it may persist for over 2 weeks when temperature is $<4^{\circ} \mathrm{C}$ (Davies et al., 2017). We estimated variation in 
indirect contacts under different decay scenarios (1, 5 and 14 days; Figure S8) and found the average indirect contact degree could increase over three times as the survival time of ASF virus increases from 1 to 5 days and the average strength can increase from 0.5 to 7.1 by increasing ASF virus survival time from 1 to 14 days, highlighting that application of our results to other study areas needs to consider the impacts of different pathogen decay rates. Given logistical constraints of the study, we attempted to monitor the largest set of animals occupying a contiguous space as possible. While we did not have a second site to use as a negative control for baits, we compared contact structure among animals with and without bait sites in their ranges (Figure S9).

We found contact and group structure of wild pigs, like racoons (Hirsch et al., 2013) and badgers (Weber et al., 2013), were dynamic across different months and sites. This variation can result from resource availability, climate conditions and reproductive cycles (Keuling et al., 2009; Mayer \& Brisbin, 2009). Thus, we may expect different management impact on contact and disease transmission in different seasons and areas, which were also suggested in fencing effects on interspecific contact and effects of climate and feeding season length in elk-brucellosis system (Cross et al., 2007; Wilber et al., 2019). Future studies with long-term active management actions might be needed to quantify the seasonality of wild pigs' response to disturbance.

\section{3 | Disease control measures}

Culling host to control diseases in wildlife populations is controversial. Where transmission is density dependent, limiting host population density below the threshold for disease invasion through culling, predation or recreational hunting should theoretically control the (re-)emergence of infectious diseases (Silk et al., 2017; Sokolow et al., 2019). Culling has been applied with varying success to control chronic wasting disease in white-tailed deer (Wild et al., 2011), bTB in badger-cattle system (Donnelly et al., 2003) and brucellosis in cattle-bison system (Schumaker et al., 2012). However, this practice can catalyse unexpected changes in host movement, like disrupting ranging or territorial behaviours, which might neutralize density impacts by increasing infectious contacts (Donnelly et al., 2003; Sokolow et al., 2019). Although previous studies have suggested significant influences of recreational hunting on spatial utilization by European wild boar (Keuling et al., 2008; Thurfjell et al., 2013), we found no detectable removal effects on wild pig contact network, likely because our removal intensity was low. Even after large perturbations like translocation, wild pigs return to pre-translocation movement patterns within several weeks (J. Smith, D. Keiter, S. Sweeney, R. Miller, P. Schlichting, \& J. Beasley, unpublished data). Results from both our observed and simulated removal scenarios provide support for a decrease in ASF relative $R_{0}$ mediated through a low-intensity removal. The primary mechanism for reduced $R_{0}$ was a reduction in host survival rather than changes in contact degree.

Our estimation of relative $R_{0}$ uses probabilistic networks to predict variation in ASF establishment over different degree distributions during the study period. However, our population-level epidemiological model is a simple proxy for the complex transmission system. Although an individual-level relative $R_{0}$ is provided to demonstrate variation in contact and ASF transmission, infectious contact rates between each animal pair (Ryder et al., 2007) and the shedding rate of ASF virus were not included in the derivation of $R_{0}$. A more detailed transmission model that accounts for additional heterogeneity in the contact network would be necessary to determine the full effects of wild pig contact dynamics on invasion of diseases like ASF for guiding optimal control strategies. Contact duration is also important for disease transmission, but we focused on contact frequency for defining network strength so that our results are comparable to those from GPS data (which do not capture contact duration). Also, given our pre-field verification in the laboratory, proximity loggers had high error rates measuring contact duration while measures of contact frequency are more accurate.

As with all empirical studies of contact structure, our results describe contact network for a limited sample across two populations (48 animals). In wildlife movement studies, this level of sampling is generally thought to be sufficient to gain general population insights; previous research found 18-58 animals was sufficient on average to capture population movement patterns in wildlife (Hebblewhite \& Haydon, 2010). Additionally, by inferring pairwise contacts probabilistically from the empirical data to understand mechanisms that drive contacts, our models can allow probabilistic prediction for larger populations. However, we estimated an approximately linear relationship between host density and average degree (Supporting Information 4; Figure S10), despite accounting for spatial limitations in contact structure. We expected this relationship would saturate at higher densities because contact structure was not homogenous in our system. The fact that we predicted saturation only weakly (upper limit of uncertainty) at higher densities suggests that our ability to predict contact structure in denser populations was constrained by our sample size. A larger sample size would improve prediction of average degree outside the host density conditions in our study. But, the fact that the upper bound on uncertainty was saturating suggests that our sample size is close to adequate for enabling outof-sample prediction. Validation of these predictions using data from denser populations to test for density-dependent effects on degree would be valuable for inferring contact structure in larger or smaller (i.e. effects of removal) populations. In conclusion, the dynamic nature of wild pig contact and high sensitivity of $R_{0}$ to contact degree highlights the importance of understanding contact patterns for estimating disease invasion risk and identifying optimal control strategies.

\section{ACKNOWLEDGEMENTS}

The logistical and financial support of the research was supplied by the USDA-APHIS National Feral Swine Damage Management Program. Field work at SRS was partially supported by the US DOE under Award Number DE-EM0004391 to the UGA Research Foundation. We thank ABIR for allowing this work to be performed 
upon the property. We are grateful for the assistance from the Savannah River Ecology Laboratory at UGA and the Range Cattle Research and Education Center at UF for data collection. We appreciate two anonymous reviewers for helpful comments.

\section{AUTHORS' CONTRIBUTIONS}

A.Y., M.Q.W., R.S.M., J.C.B., R.K.B., K.C.V., G.W. and K.M.P. designed the study; P.S., B.W., W.M.A., S.M.C., J.C.B. and R.K.B. collected field data; A.Y. implemented the analyses and wrote the first draft of the manuscript. All authors contributed to manuscript writing, editing and gave final approval for publication.

\section{DATA AVAILABILITY STATEMENT}

Data are available via the Dryad Digital Repository https://doi.org/ 10.5061/dryad.0p2ngf205 (Yang et al., 2020).

\section{ORCID}

Anni Yang (iD https://orcid.org/0000-0002-9535-2193

Mark Q. Wilber iD https://orcid.org/0000-0002-8274-8025

Ryan S. Miller iD https://orcid.org/0000-0003-3892-0251

James C. Beasley iD https://orcid.org/0000-0001-9707-3713

George Wittemyer (iD https://orcid.org/0000-0003-1640-5355

Kim M. Pepin (iD https://orcid.org/0000-0002-9931-8312

\section{REFERENCES}

Bansal, S., Grenfell, B. T., \& Meyers, L. A. (2007). When individual behaviour matters: Homogeneous and network models in epidemiology. Journal of the Royal Society Interface, 4(16), 879-891. https://doi. org/10.1098/rsif.2007.1100

Bastille-Rousseau, G., Schlichting, P., Keiter, D. A., Smith, J., Kilgo, J. C., Wittemyer, G., Vercauteren, K., \& Beasley, J. C., \& Pepin, K. M. (2020). Multi-level movement response of invasive wild pigs (Sus scrofa) to removal. Pest Management Science. Pest Management Science, https://doi.org/10.1002/ps.6029

Bates, T. W., Thurmond, M. C., \& Carpenter, T. E. (2003). Description of an epidemic simulation model for use in evaluating strategies to control an outbreak of foot-and-mouth disease. American Journal of Veterinary Research, 64(2), 195-204. https://doi.org/10.2460/ ajvr.2003.64.195

Beasley, J. C., Ditchkoff, S. S., Mayer, J. J., Smith, M. D., \& Vercauteren, K. C. (2018). Research priorities for managing invasive wild pigs in North America. The Journal of Wildlife Management, 82(4), 674-681. https://doi.org/10.1002/jwmg.21436

Best, E. C., Dwyer, R. G., Seddon, J. M., \& Goldizen, A. W. (2014). Associations are more strongly correlated with space use than kinship in female eastern grey kangaroos. Animal Behaviour, 89, 1-10. https://doi.org/10.1016/j.anbehav.2013.12.011

Boadella, M., Vicente, J., Ruiz-Fons, F., De la Fuente, J., \& Gortázar, C. (2012). Effects of culling Eurasian wild boar on the prevalence of Mycobacterium bovis and Aujeszky's disease virus. Preventive Veterinary Medicine, 107(3-4), 214-221. https://doi.org/10.1016/j. prevetmed.2012.06.001

Boehm, M., Hutchings, M. R., \& White, P. C. (2009). Contact networks in a wildlife-livestock host community: Identifying highrisk individuals in the transmission of bovine TB among badgers and cattle. PLoS ONE, 4(4), e5016. https://doi.org/10.1371/journal. pone.0005016

Brahmbhatt, D. P., Fosgate, G. T., Dyason, E., Budke, C. M., Gummow, B., Jori, F., Ward, M. P., \& Srinivasan, R. (2012). Contacts between domestic livestock and wildlife at the Kruger National Park Interface of the Republic of South Africa. Preventive Veterinary Medicine, 103(1), 16-21. https://doi.org/10.1016/j.prevetmed.2011.08.003

Cowled, B., \& Garner, G. (2008). A review of geospatial and ecological factors affecting disease spread in wild pigs: Considerations for models of foot-and-mouth disease spread. Preventive Veterinary Medicine, 87(3-4), 197-212. https://doi.org/10.1016/j.prevetmed. 2008.03.012

Craft, M. E. (2015). Infectious disease transmission and contact networks in wildlife and livestock. Philosophical Transactions of the Royal Society B: Biological Sciences, 370(1669), 20140107. https://doi.org/10.1098/ rstb.2014.0107

Cross, P. C., Edwards, W. H., Scurlock, B. M., Maichak, E. J., \& Rogerson, J. D. (2007). Effects of management and climate on elk brucellosis in the Greater Yellowstone Ecosystem. Ecological Applications, 17(4), 957-964. https://doi.org/10.1890/06-1603

Crowl, T. A., Crist, T. O., Parmenter, R. R., Belovsky, G., \& Lugo, A. E. (2008). The spread of invasive species and infectious disease as drivers of ecosystem change. Frontiers in Ecology and the Environment, 6(5), 238-246. https://doi.org/10.1890/070151

Dardaillon, M. (1988). Wild boar social groupings and their seasonal changes in the Camargue, southern France. Zeitschrift Für Säugetierkunde, 53(1), 22-30.

Davies, K., Goatley, L., Guinat, C., Netherton, C., Gubbins, S., Dixon, L., $\&$ Reis, A. (2017). Survival of African swine fever virus in excretions from pigs experimentally infected with the Georgia 2007/1 isolate. Transboundary and Emerging Diseases, 64(2), 425-431. https://doi. org/10.1111/tbed.12381

Dietz, K. (1993). The estimation of the basic reproduction number for infectious diseases. Statistical Methods in Medical Research, 2(1), 23-41. https://doi.org/10.1177/096228029300200103

Donnelly, C. A., Woodroffe, R., Cox, D., Bourne, J., Gettinby, G., Le Fevre, A. M., McInerney, J. P., \& Morrison, W. I. (2003). Impact of localized badger culling on tuberculosis incidence in British cattle. Nature, 426(6968), 834-837.

Drewe, J. A., Eames, K. T., Madden, J. R., \& Pearce, G. P. (2011). Integrating contact network structure into tuberculosis epidemiology in meerkats in South Africa: Implications for control. Preventive Veterinary Medicine, 101(1-2), 113-120. https://doi.org/10.1016/ j.prevetmed.2011.05.006

Drewe, J. A., Weber, N., Carter, S. P., Bearhop, S., Harrison, X. A., Dall, S. R. X., McDonald, R. A., \& Delahay, R. J. (2012). Performance of proximity loggers in recording intra-and inter-species interactions: $A$ laboratory and field-based validation study. PLOS ONE, 7(6), e39068. https://doi.org/10.1371/journal.pone.0039068

Fattebert, J., Baubet, E., Slotow, R., \& Fischer, C. (2017). Landscape effects on wild boar home range size under contrasting harvest regimes in a human-dominated agro-ecosystem. European Journal of Wildlife Research, 63(2), 32. https://doi.org/10.1007/s1034 4-017-1090-9

Gabor, T. M., Hellgren, E. C., Van Den Bussche, R. A., \& Silvy, N. J. (1999). Demography, sociospatial behaviour and genetics of feral pigs (Sus scrofa) in a semi-arid environment. Journal of Zoology, 247(3), 311322. https://doi.org/10.1111/j.1469-7998.1999.tb00994.x

Gelman, A., Hwang, J., \& Vehtari, A. (2014). Understanding predictive information criteria for Bayesian models. Statistics and Computing, 24(6), 997-1016. https://doi.org/10.1007/s11222-01 3-9416-2

Gelman, A., \& Rubin, D. B. (1992). Inference from iterative simulation using multiple sequences. Statistical Science, 7(4), 457-472. https:// doi.org/10.1214/ss/1177011136

Getz, W. M., Fortmann-Roe, S., Cross, P. C., Lyons, A. J., Ryan, S. J., \& Wilmers, C. C. (2007). LoCoH: Nonparameteric kernel methods for constructing home ranges and utilization distributions. PLoS ONE, 2(2), e207. https://doi.org/10.1371/journal.pone.0000207 
Girvan, M., \& Newman, M. E. (2002). Community structure in social and biological networks. Proceedings of the National Academy of Sciences of the United States of America, 99(12), 7821-7826. https://doi.org/ 10.1073/pnas.122653799

Grear, D. A., Samuel, M. D., Scribner, K. T., Weckworth, B. V., \& Langenberg, J. A. (2010). Influence of genetic relatedness and spatial proximity on chronic wasting disease infection among female whitetailed deer. Journal of Applied Ecology, 47(3), 532-540. https://doi. org/10.1111/j.1365-2664.2010.01813.x

Hebblewhite, M., \& Haydon, D. T. (2010). Distinguishing technology from biology: A critical review of the use of GPS telemetry data in ecology. Philosophical Transactions of the Royal Society B: Biological Sciences, 365(1550), 2303-2312.

Hirsch, B. T., Prange, S., Hauver, S. A., \& Gehrt, S. D. (2013). Raccoon social networks and the potential for disease transmission. PLoS ONE, 8(10), e75830. https://doi.org/10.1371/journal.pone.0075830

Imm, D., \& McLeod, K. (2005). Plant communities. In J. Kilgo (Ed.), Ecology and management of a forested landscape: Fifty years on the Savannah River site (pp. 106-161). Island Press.

Keiter, D. A., Davis, A. J., Rhodes, O. E., Cunningham, F. L., Kilgo, J. C., Pepin, K. M., \& Beasley, J. C. (2017). Effects of scale of movement, detection probability, and true population density on common methods of estimating population density. Scientific Reports, 7(1), 1-12. https://doi.org/10.1038/s41598-017-09746-5

Kellner, K. (2015). JagsUI: A wrapper around rjags to streamline JAGS analyses. R Package Version 1.5.1. Retrieved from https://cran.r-project. org/web/packages/jagsUl/index.html

Keuling, O., Stier, N., \& Roth, M. (2008). How does hunting influence activity and spatial usage in wild boar Sus scrofa L.? European Journal of Wildlife Research, 54(4), 729. https://doi.org/10.1007/s10344-0080204-9

Keuling, O., Stier, N., \& Roth, M. (2009). Commuting, shifting or remaining? Different spatial utilisation patterns of wild boar Sus scrofa L. in forest and field crops during summer. Mammalian Biology, 74(2), 145-152.

Lyons, A. J. (2014). T-LoCoH tips: Analyzing isopleth overlap. Retrieved from Tlocoh.r-forge.r-project.org/; http://tlocoh.r-forge.r-project.org/ tips/isopleth_overlap.html

Lyons, A. J., Turner, W. C., \& Getz, W. M. (2013). Home range plus: A space-time characterization of movement over real landscapes. Movement Ecology, 1(1), 2. https://doi.org/10.1186/2051-3933-1-2

Mayer, J., \& Brisbin, L. (2009). Wild pigs: Biology, damage, control techniques and management (No. SRNL-RP-2009-00869; p. 408). Savannah River National Laboratory.

McDowell, A. (2003). From the help desk: Hurdle models. The Stata Journal, 3(2), 178-184. https://doi.org/10.1177/1536867X03003 00207

Miller, R. S., Sweeney, S. J., Slootmaker, C., Grear, D. A., Di Salvo, P. A., Kiser, D., \& Shwiff, S. A. (2017). Cross-species transmission potential between wild pigs, livestock, poultry, wildlife, and humans: Implications for disease risk management in North America. Scientific Reports, 7(1), 1-14. https://doi.org/10.1038/s41598-01707336-z

Pepin, K. M., Davis, A. J., Beasley, J., Boughton, R., Campbell, T., Cooper, S. M., Gaston, W., Hartley, S., Kilgo, J. C., Wisely, S. M., Wyckoff, C., \& VerCauteren, K. C. (2016). Contact heterogeneities in feral swine: Implications for disease management and future research. Ecosphere, 7(3), e01230. https://doi.org/10.1002/ecs2.1230

Plummer, M. (2003). JAGS: A program for analysis of Bayesian graphical models using Gibbs sampling. In Proceedings of the 3rd international workshop on distributed statistical, Vol. 124(125), pp. 1-10.

Podgórski, T., Apollonio, M., \& Keuling, O. (2018). Contact rates in wild boar populations: Implications for disease transmission. The Journal of Wildlife Management, 82(6), 1210-1218. https://doi.org/10.1002/ jwmg. 21480
Podgórski, T., Lusseau, D., Scandura, M., Sönnichsen, L., \& Jędrzejewska, B. (2014). Long-lasting, kin-directed female interactions in a spatially structured wild boar social network. PLoS ONE, 9(6), e99875. https:// doi.org/10.1371/journal.pone.0099875

R Core Team. (2019). R: A language and environment for statistical computing. R Foundation for Statistical Computing. Retrieved from https:// www.R-project.org/

Reynolds, J. J., Hirsch, B. T., Gehrt, S. D., \& Craft, M. E. (2015). Raccoon contact networks predict seasonal susceptibility to rabies outbreaks and limitations of vaccination. Journal of Animal Ecology, 84(6), 17201731. https://doi.org/10.1111/1365-2656.12422

Ryan, S. J., Jones, J. H., \& Dobson, A. P. (2013). Interactions between social structure, demography, and transmission determine disease persistence in primates. PLoS ONE, 8(10), e76863. https://doi.org/ 10.1371/journal.pone.0076863

Ryder, J. J., Miller, M. R., White, A., Knell, R. J., \& Boots, M. (2007). Hostparasite population dynamics under combined frequency-and density-dependent transmission. Oikos, 116(12), 2017-2026. https://doi. org/10.1111/j.2007.0030-1299.15863.x

Sah, P., Mann, J., \& Bansal, S. (2018). Disease implications of animal social network structure: A synthesis across social systems. Journal of Animal Ecology, 87(3), 546-558. https://doi.org/10.1111/13652656.12786

Sánchez-Vizcaíno, J. M., Mur, L., \& Martínez-López, B. (2013). African swine fever (ASF): Five years around Europe. Veterinary Microbiology, 165(1-2), 45-50. https://doi.org/10.1016/j.vetmic.2012.11.030

Schumaker, B. A., Peck, D. E., \& Kauffman, M. E. (2012). Brucellosis in the Greater Yellowstone area: Disease management at the wildlife-livestock interface. Human-Wildlife Interactions, 6(1), 7.

Silk, M. J., Croft, D. P., Delahay, R. J., Hodgson, D. J., Boots, M., Weber, N., \& McDonald, R. A. (2017). Using social network measures in wildlife disease ecology, epidemiology, and management. BioScience, 67(3), 245-257. https://doi.org/10.1093/biosci/biw175

Snow, N. P., Lavelle, M. J., Halseth, J. M., Glow, M. P., VanNatta, E. H., Davis, A. J., Pepin, K. M., Tabor, R. T., Leland, B. R., Staples, L. D., \& VerCauteren, K. C. (2019). Exposure of a population of invasive wild pigs to simulated toxic bait containing biomarker: Implications for population reduction. Pest Management Science, 75(4), 1140-1149. https://doi.org/10.1002/ps.5235

Sokolow, S. H., Nova, N., Pepin, K. M., Peel, A. J., Pulliam, J. R. C., Manlove, K., Cross, P. C., Becker, D. J., Plowright, R. K., McCallum, H., \& De Leo, G. A. (2019). Ecological interventions to prevent and manage zoonotic pathogen spillover. Philosophical Transactions of the Royal Society B: Biological Sciences, 374(1782), 20180342. https://doi. org/10.1098/rstb.2018.0342

Swain, H. M., Boughton, E. H., Bohlen, P. J., \& Lollis, L. O. (2013). Tradeoffs among ecosystem services and disservices on a Florida ranch. Rangelands, 35(5), 75-87. https://doi.org/10.2111/RANGELANDS-D13-00053.1

Thurfjell, H., Spong, G., \& Ericsson, G. (2013). Effects of hunting on wild boar Sus scrofa behaviour. Wildlife Biology, 19(1), 87-93.

Vatcheva, K. P., Lee, M., McCormick, J. B., \& Rahbar, M. H. (2016). Multicollinearity in regression analyses conducted in epidemiologic studies. Epidemiology (Sunnyvale, Calif.), 6(2). https://doi.org/ 10.4172/2161-1165.1000227

VerCauteren, K., Beasley, J., Ditchkoff, S. S., Mayer, J. J., Roloff, G. J., \& Strickland, B. K. (2020). Invasive wild pigs in North America: Ecology, Impacts, and Management. CRC Press.

Weber, N., Carter, S. P., Dall, S. R., Delahay, R. J., McDonald, J. L., Bearhop, S., \& McDonald, R. A. (2013). Badger social networks correlate with tuberculosis infection. Current Biology, 23(20), R915-R916. https:// doi.org/10.1016/j.cub.2013.09.011

Welch, D., Bansal, S., \& Hunter, D. R. (2011). Statistical inference to advance network models in epidemiology. Epidemics, 3(1), 38-45. https://doi.org/10.1016/j.epidem.2011.01.002 
White, L. A., Forester, J. D., \& Craft, M. E. (2017). Using contact networks to explore mechanisms of parasite transmission in wildlife. Biological Reviews, 92(1), 389-409. https://doi.org/10.1111/brv.12236

Wilber, M. Q., Pepin, K. M., Campa, H., Hygnstrom, S. E., Lavelle, M. J., Xifara, T., VerCauteren, K. C., \& Webb, C. T. (2019). Modelling multi-species and multi-mode contact networks: Implications for persistence of bovine tuberculosis at the wildlife-livestock interface. Journal of Applied Ecology, 56(6), 1471-1481. https://doi.org/10.1111/ 1365-2664.13370

Wild, M. A., Hobbs, N. T., Graham, M. S., \& Miller, M. W. (2011). The role of predation in disease control: A comparison of selective and nonselective removal on prion disease dynamics in deer. Journal of Wildlife Diseases, 47(1), 78-93. https://doi.org/10.7589/ 0090-3558-47.1.78

Yang, A., Schlichting, P., Wight, B., Anderson, W. M., Chinn, S. M., Wilber, M. Q., Miller, R. S., Beasley, J. C., Boughton, R. K., VerCauteren, K. C., Wittemyer, G., \& Pepin, K. M. (2020). Data from: Effects of social structure and management on risk of disease establishment in wild pigs. Dryad Digital Repository, https://doi.org/10.5061/dryad.0p2ng f205

\section{SUPPORTING INFORMATION}

Additional supporting information may be found online in the Supporting Information section.

How to cite this article: Yang A, Schlichting P, Wight B, et al. Effects of social structure and management on risk of disease establishment in wild pigs. J Anim Ecol. 2021;90:820-833. https://doi.org/10.1111/1365-2656.13412 